\title{
Radiofrequency catheter ablation of ventricular tachycardia in structural heart disease: single team experience with follow-up upto 5 years
}

Introduction: Catheter ablation of ventricular tachycardia (VT) in structural heart disease has significantly evolved over the past few decades. We present our experience of VT ablation in such patients over last five years.

Methods and Results: Out of 38 cases of VT ablation in patients with structural heart disease done during last five years, 18 had chronic myocardial scar, 2 had recent myocardial infarction, 1 dilated cardiomyopathy and 17 ARVD/C. Substrate modifications was performed as first step in majority of patients by using 3D electroanatomical system ( $N a v X$, St. Jude Medical, USA). VT induction was tried after substrate modification. End point of ablation was non-inducibility of all VTs. Failure of RFA was seen in no patient. Partial success of RFA was seen in 2 and in $36(95 \%)$ the procedure was fully successful. Fifteen patients already had an ICD implanted whereas 20 patients underwent ICD implantation after the procedure. Median Follow-up available is for 38 months. Four patients had recurrence of VT identified by the ICD. No patient had sudden cardiac death. One patient of ARVD/C had progressive RV failure.

Conclusion: VT ablation in patients with underlying structural heart disease significantly reduces the recurrence of VT episodes. It plays an important role in reducing the number of ICD therapies.

Submitted: 28 December 2015; Accepted: 09 January 2016; Published online: 15 January 2016

Keywords: ARVD/C - Radiofrequency ablation - Electroanatomical mapping - Ventricular tachycardia - Structural heart disease - Cardiomyopathy.

Radiofrequency catheter ablation (RFA) of ventricular tachycardia (VT) has significantly evolved over the past few decades. Ablation of idiopathic VT in the absence of structural heart disease is now well accepted as a safe and effective procedure. In contrast, ablation of VT with underlying structural heart disease is more challenging [1], mainly because of the heterogeneous reentrant circuits involved and the location of the circuit deep inside the scar. Experience and understanding about VT in structural heart disease has increased over last few years. From the fundamental understanding of the reentrant mechanism in scar-mediated VT to the advent and routine implementation of electroanatomic mapping, catheter ablation has emerged from a palliative last-resort therapy to a more preemptive strategy for patients at risk for arrhythmia recurrence [2].
Vikas Kataria', Amitabh Yaduvanshi \& Mohan Nair ${ }^{3 *}$ 'Department of Cardiology, Senior Consultant, Holy Family Hospital, New Delhi

2Department of Cardiology, Senior Consultant, Holy Family Hospital, New Delhi

${ }^{3}$ Department of Cardiology, Holy Family Hospital, New Delhi *Author for correspondence: Tel.: +919910014371 mohancardio@gmail.com 
We report the acute and intermediate-term results of RFA of ventricular tachycardia in patients with a spectrum of structural heart disease.

\section{Methods}

Between 1st April 2010 and 31st March 2015, all patients who underwent RFA for ventricular tachycardia and had underlying structural heart disease were included in this prospective analysis. Each patient had previously experienced one or more episodes of documented sustained VT.

All patients gave written informed consent for RFA. Before the ablation catheter was placed in left ventricle, heparin was given to achieve an activated clotting time $>250$ s. Substrate modification was the preferred first strategy for ablation in majority of the patient. Activation map was used as first strategy only in patients with hemodynamically stable sustained VT and/ or those in whom a well- defined substrate map could not be obtained(ARVC/D).Irrigated tip ablation catheter was used in most of the patients except for few patients in the initial part of the study where non-irrigated catheters were used for RFA. For irrigated tip catheters, the settings were: $60 \mathrm{~W}, 400 \mathrm{c}$ for $60 \mathrm{~s}, 15$ $\mathrm{mL} / \mathrm{min}$ flow; for non- irrigated tip catheters, the RF settings were: $60 \mathrm{~W}, 600 \mathrm{c}$ for $60 \mathrm{~s}$. Ablation at any point was considered adequate when there was a minimum of $50 \%$ decrease in the intracardiac electrogram voltage. Detailed mapping was done, under 3D electroanatomical guidance (NavX, St. Jude Medical, St. Paul, MN) to delineate scars.(Scar was defined as area showing $<0.5 \mathrm{mv}$ on voltage map). Once the scar was defined, scar homogenization was done by either a) small scars were isolated from the surrounding healthy myocardium by RF ablation (Figure1A) or b) in case of large scars, careful delineation and ablation of channels of healthy tissue within or around scars was targeted (Figure 1B). VT induction was attempted only after substrate modification. Ablation was first performed during sinus rhythm; the inducibility of VT was assessed by programmed electrical stimulation with one to three extra stimuli at two sites. Ablation during VT was done only if a stable VT was induced after completion of step 1. For ablation during VT, termination of the tachycardia during energy application was an essential end point. Goal of the ablation was non-inducibility of all VTs. If a VT could be induced after initial ablation, then further ablation was done using substrate map or activation map according to the hemodynamic stability/ sustainability of VT. EnSite Array (St. Jude Medical, St. Paul, MN) mapping system was used in case of VTs originating from RV which were hemodynamically unstable or non- sustained, particularly in patients with ARVC/D (Figure 2). Using this mapping and ablation technique, we rarely had to go back to the same point for repeat energy delivery.

Ensite Array system creates three-dimensional electroanatomical maps without the need for point-bypoint contact electrograms. In this system, non-contact balloon catheter creates virtual unipolar electrograms using a mathematical inverse solution to estimate the electrical potentials which allows for non-contact mapping of conduction through a cardiac chamber in a single cycle of the tachycardia. This system is particularly useful for mapping and ablation of VT which are nonsustained and/or hemodynamically unstable.

We also used rotational angiography (Dyna CTSiemens, Erlangen, Germany), for accurate realtime anatomic and volume data. Fusion of rotational angiographic image and NavX map improved the anatomic accuracy of the chambers (Figure 3).

Clinical VT(s) were defined by comparison of 12lead electrocardiographic morphologies of the available ECGs with induced VT. When12-lead ECG of the clinical VT was not available; we matched the cycle lengths of induced and spontaneous VT.

Procedure was defined as successful if all of the inducible VTs could be successfully mapped and ablated, partially successful when not all of the inducible VTs were successfully ablated, but the clinical VT could be ablated successfully, and failure if none of the inducible VTs could be ablated successfully. Longterm success was defined as the absence of recurrence of VT over follow-up.

Follow-up: Patients were followed up in outpatient clinics; the patient underwent monthly 24-hr Holter monitoring for 6 months and then every three months. Patients were advised to report any symptom of palpitation or syncope/presyncope. They were also advised to get a12-lead ECG in case of such symptoms. AICD interrogation reports were analyzed whenever available. VT recurrence was defined as an appropriate AICD intervention or the occurrence of sustained VT of at least $30 \mathrm{~s}$ duration or any hemodynamically significant VT subsequent to the ablation procedure as documented by a 12-lead electrocardiogram, an event monitor, or a 24-hr Holter monitor. The time to VT recurrence after each ablation procedure was noted. Long- term success was defined as the absence of recurrence of VT over follow-up.

\section{Results}

Out of 124 cases of VT ablation performed between 1st April 2010 and 31st March 2015, 38 cases had underlying structural heart disease. Mean age of the patients was $43.9 \pm 10.6$ years and $58 \%$ were males. Baseline characteristics of these patients are given in table 1 . Of the 38 cases, 17 (45\%) had ARVD/C, 

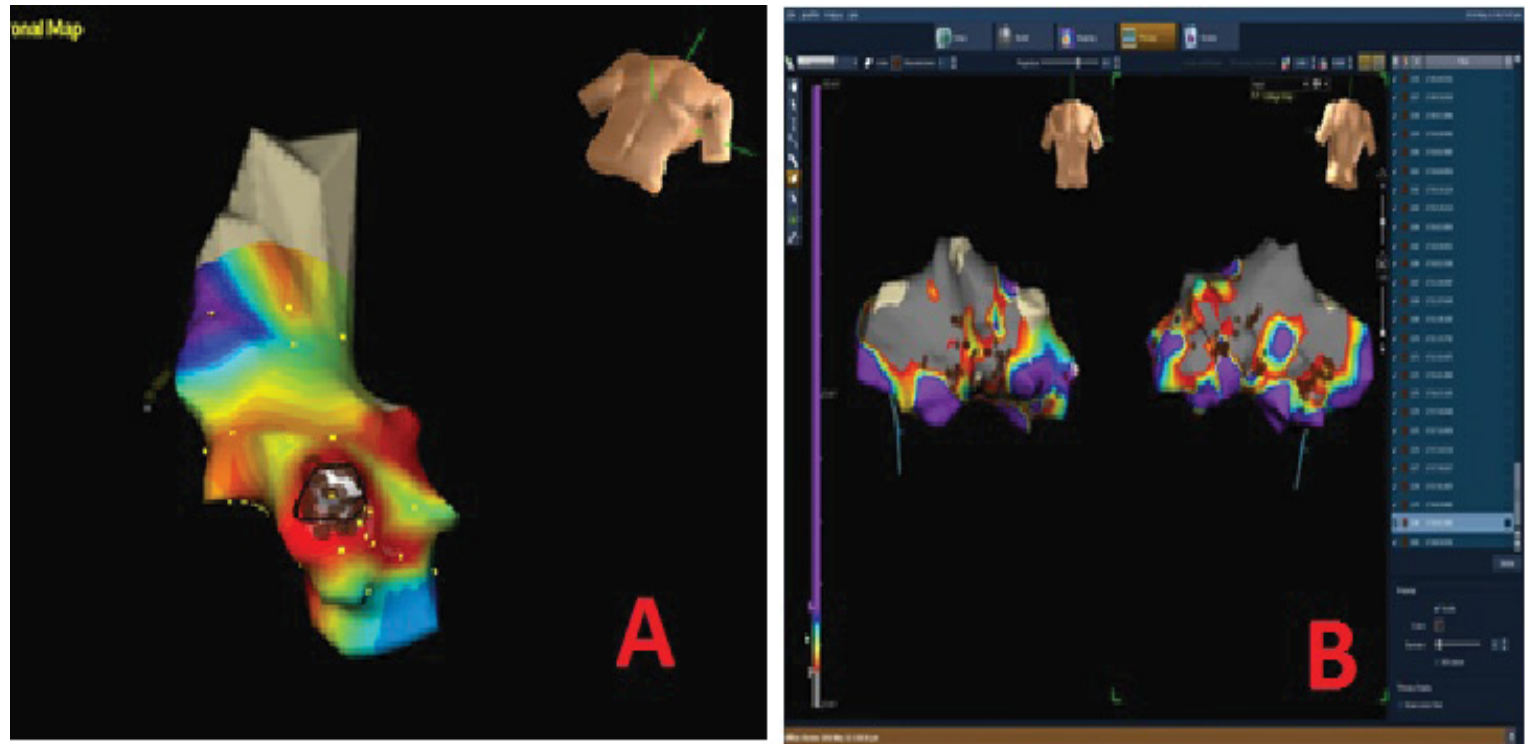

Figure 1: (A) Electroanatomical map from a patient with small scar (White arrow) on the antero-apical region. Small scars were isolated from the rest of the myocardium by the RF lesions (Brown dots). (B) Electroanatomical map from a patient with large scar (white star). In such scars, channels of healthy myocardium (white arrow) were carefully delineated and ablated.

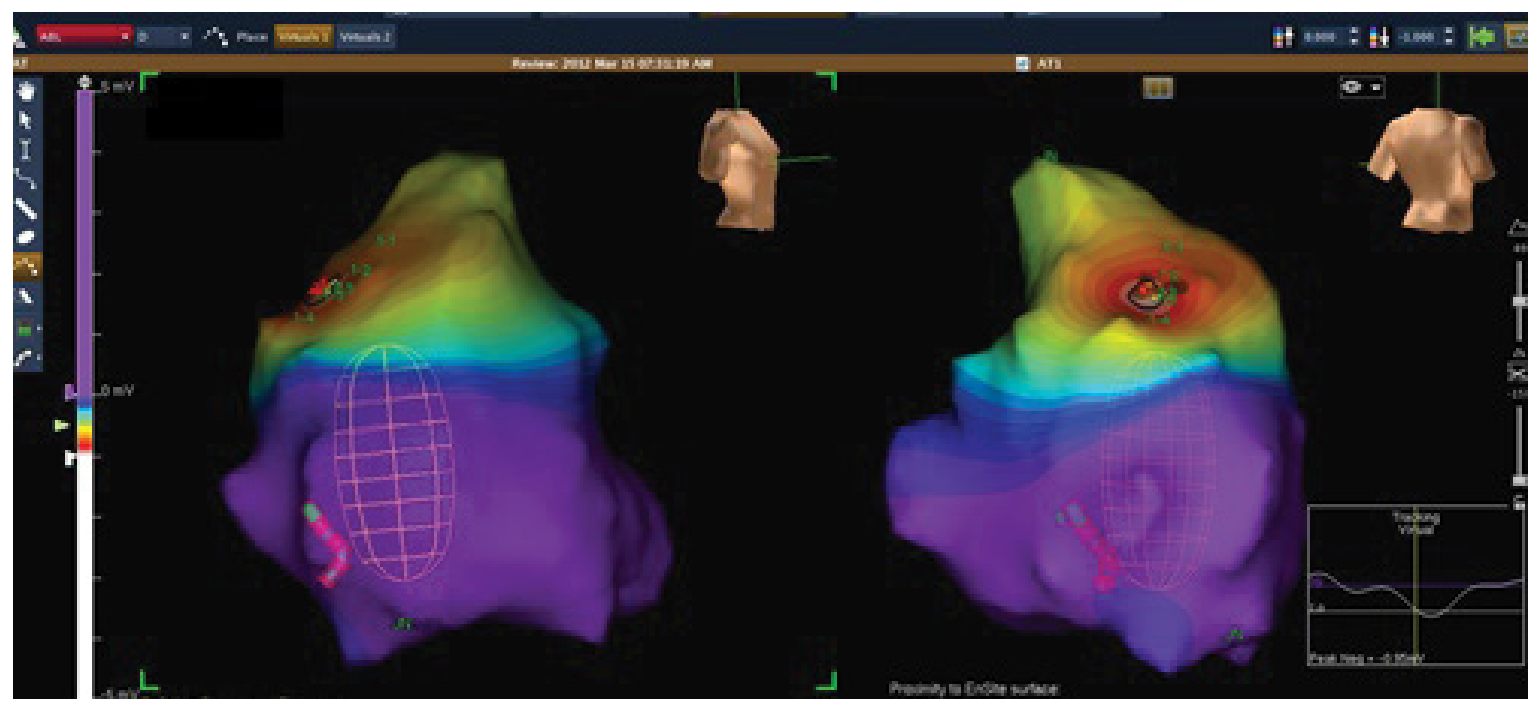

Figure 2: Activation map created by using non-contact mapping (Ensite Array, arrows).

$18(47 \%)$ had underlying chronic scar, $2(5 \%)$ had recent myocardial infarction and 1 (3\%) had dilated cardiomyopathy (DCMP).

\section{Electrophysiology study, mapping, and ablation characteristics}

Substrate modification was used as initial first strategy in majority of the patients $(89.5 \%$, Table 1$)$. Four patients $(10.5 \%)$ had hemodynamically stable, sustained VT allowing activation mapping as initial first step.

VT induction was performed after substrate modification. Twenty patients had inducible VT, with multiple VT morphology induced in eight patients.
In four patients, the induced VT was non-sustained but the short duration was enough to get relevant information on the noncontact map for guiding ablation. In two patients, VT induced after ablation of the clinical VT caused hemodynamic collapse requiring $\mathrm{DC}$ cardioversion; however, the short run of VT prior to cardioversion was sufficient to map the tachycardia (Figure 2). Two patients of ARVD/C required ablation through epicardial route. This was done after extensive ablation endocardially failed to abolish clinically important VT. The technique used for Epicardial ablation was as described by Sosa E et al. [3].

All VTs could be ablated successfully in 36 (95\%) patients. Only clinical VTs could be ablated in two 


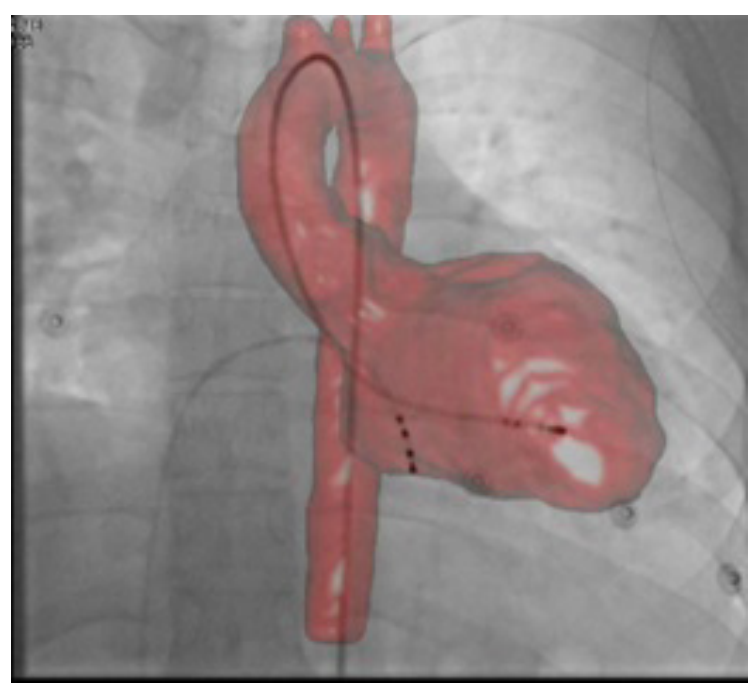

Figure 3: Fusion of rotational angiographic image (red colored) over live fluoroscopic image during RFA in a patient with LV apical aneurysm (arrow) and ventricular tachycardia.

patients (partial success $=5 \%$ ). None of the patient had failure of RFA (Table 1). There was no periprocedural complication. All patients were discharged from the hospital 48 hours after the procedure.

Fifteen patients already had an ICD implanted before the RFA whereas 20 patients got an ICD implanted after the procedure. Three patients refused for ICD implantation. All patients were advised an ICD as a) The recurrence rates following RF ablation as a standalone procedure in these patients was not known and b) RFA is not yet recommended as destination therapy for these patients.

\section{Follow up}

Median follow-up was 38 (24 to 60) months. No sudden cardiac death was observed in any of the patient. No episode of sustained VT was observed on routine monthly Holter recordings of any patient. All patients with an ICD had systematic interrogation of the device at each visit, to look for any arrhythmia since the last visit. Four patients had recurrence of VT which was picked up during ICD interrogation. One patient had a recurrence of slow VT at the rate of 146 beats per minute, after 17 weeks of ablation which was clinically silent and was picked up on ICD interrogation and responded to anti-tachycardia pacing by the device. Three other patients received appropriate ICD therapy during 2nd, 10th and 27th month after RFA respectively. Of those four patients who had recurrence of VT, 3 had $\mathrm{ARVD} / \mathrm{C}$ as the underlying disease while one patient had DCMP. All other patients (34/38) remained VT free till the last follow-up making a long VT free success rate of $89 \%$.

Antiarrhythmic medications were discontinued if there was no recurrence of VT for 6 months. Antiarrhythmic medication could be withdrawn in all patients after 6-8 months of ablation except for those four patients who had recurrence, in whom the medication could not be stopped or had to be restarted. One patient with ARVD/C had progressive right ventricular failure.

\section{Discussion}

This study shows that catheter ablation of VT in patients with structural heart disease is associated with high acute procedural success and good intermediateterm outcomes. In their study on patient with ARVD/C, Ellison et al. [4] did not observe any recurrence of VT during a mean follow-up of 17 months after catheter ablation. However, three patients were continuing on antiarrhythmic drugs. H. Calkins et al. [1] reported an acute success rate (defined as absence of all inducible VTs) of $75 \%$ in patients with structural heart disease. But recurrence of VT after $243 \pm 153$ days (convert to months) of ablation was observed in $46 \%$ of the patients. Similar acute success rate of $74 \%$ was reported by Marchlinski et al. [5]. After a follow-up of $27 \pm$ 22 months, $89 \%$ of patients were free of recurrence. However, repeat ablation procedures were required in $68 \%$ of patients, and the use of antiarrhythmic drugs was not reported. Thus, the success rates of a single procedure were low. The results of this study are in keeping with the high acute success rate reported by other studies $[1,4,5]$. We have previously reported our results with ablation in ARVC/D [5].

Studies have reported variable recurrence rates after VT ablation. Most studies have reported higher rates than the present study. Recurrence of $40 \%$ was reported by Reithmann et al. [7] during a follow-up period of 7 months. Study by Verma et al. [6] reported $47 \%$ recurrence at 3 years of follow-up. The difference in recurrence rates compared to our study may be explained on the basis of the use of 3D mapping system. Substrate base ablation using 3D electroanatomical mapping has been used with good success rates in ablation of VT in complex substrates such as postinfarction [8] and cardiomyopathies. In the study by Dalal et al. [9], only $21 \%$ patients had ablation done using 3D electroanatomical mapping. In addition, we used rotational angiography to define real time anatomy resulting in better contact during ablation. This has not been reported previously. Moreover, most of the earlier large studies have pooled data from several centers, with recruitment rates as low as two patients from a single center, resulting in confounding, onuniform results $[6,8,9]$. Our study used the noncontact 


\begin{tabular}{|cc|}
\hline Table 1: & Baseline, procedural characteristics and outcomes. \\
\hline Total number & 38 \\
\hline Age (Years) & $43.9 \pm 10.6$ \\
\hline Male (\%) & 58 \\
\hline ARVD/C & $17(45 \%)$ \\
\hline Post MI Scar & $18(47 \%)$ \\
\hline Recent MI & $2(5 \%)$ \\
\hline DCMP & $1(2.6 \%)$ \\
\hline Initial step: Substrate map & $34(89.5 \%)$ \\
\hline Initial step: Activation map & $4(10.5 \%)$ \\
\hline Epicardial ( or combined) Ablation & $2(5 \%)$ \\
\hline Endocardial Ablation & $36(95 \%)$ \\
\hline ICD (before RFA) & $15(39 \%)$ \\
\hline ICD (after RFA) & $20(53 \%)$ \\
\hline Successful RFA & $36(95 \%)$ \\
\hline Partial success & $2(5 \%)$ \\
\hline Failure of RFA & $0(0 \%)$ \\
\hline Recurrence & $4(11 \%)$ \\
\hline Long term success & $34(89 \%)$ \\
\hline
\end{tabular}

mapping system (for patients with non-sustained or hemodynamically unstable VT) which has a potential advantage in this setting. This is in agreement with the study by Yao et al. [10] who used non-contact mapping in similar subset of patients.

\section{Limitations}

The study included a population of patients with varied structural heart disease, even though the maximum patients were of post- infarction scar.

More recent techniques such as scar homogenization were not routinely used in our patients.

\section{Conclusion}

VT ablation in patients with structural heart disease has high acute success rate and low intermediate term recurrence rate. It is an effective tool in management of such patients and effective in reducing ICD therapies.

\section{Executive summary}

- We report here a case of central sleep apnea syndrome associated with frequent nocturnal premature ventricular contractions (PVCs) that were successfully eliminated using nocturnal oxygen therapy.

- This case report suggests the possibility of an impact of oxygen inhalation during sleep in patients with central SAS on the prevention of ventricular arrhythmias during both the daytime and night. However, further wellestablished randomized, controlled studies are needed to confirm these effects.

\section{References}

1. Calkins H, Epstein A, Packer D, et al. Catheter Ablation of Ventricular Tachycardia in Patients With Structural Heart Disease Using Cooled Radiofrequency Energy. J. Am. Coll. Cardiol. 35, 1905-1914 (2000).

2. Reddy VY, Reynolds MR, Neuzil P, et al. Prophylactic catheter ablation for the prevention of defibrillator therapy. $N$. Engl. J. Med. 357, 2657-2665 (2007).

3. Sosa E, Scanavacca M, D'Avila A, Bellotti G, Pilleggi F. Radiofrequency Catheter Ablation of Ventricular Tachycardia Guided by Nonsurgical Epicardial Mapping in Chronic Chagasic Heart Disease. Pacing and Clinical Electrophysiology 22, 128-130 (1999).

4. Ellison KE, Friedman PL, Ganz L, Stevenson WG. Entrainment mapping and radiofrequency catheter ablation of ventricular tachycardia in right ventricular dysplasia. J. Am. Coll. Cardiol. 32, 724-728 (1998).

5. Marchlinski FE, Zado E, Dixit S, et al. Electroanatomical substrate and outcome of catheter ablative therapy for ventricular tachycardia in setting of right ventricular cardiomyopathy. Circulation. 110, 2293-2298 (2004).

6. Nair M, Yaduvanshi A, Kataria V, Kumar M. Radiofrequency catheter ablation of ventricular tachycardia in arrhythmogenic rightventricular dysplasia/cardiomyopathy using non-contact electroanatomical mapping: single-center experience with follow-up up to median of 30 months. $J$. Interv. Card. Electrophysiol. 31, 141-147 (2011).

7. Reithmann C, Hahnefeld A, Remp T, et al. Electroanatomical mapping of endocardial right ventricular activation as a guide for catheter ablation in patients with arrhythmogenic right ventricular dysplasia. Pacing and Clinical Electrophysiology 26, 1308-1316 (2003).

8. Verma A, Kilicaslan F, Schweikert RA, et al. Short- and long-term success of substrate-based mapping and ablation of ventricular tachycardia in arrhythmogenic right ventricular dysplasia. Circulation. 111, 3209-3216 (2005).

9. Verma A, Kilicaslan F, Schweikert RA, Tomassoni G, Rossillo A. Long-Term Efficacy of Catheter Ablation of Ventricular Tachycardia in Patients With Arrhythmogenic Right Ventricular Dysplasia/ Cardiomyopathy. J. Am. Coll. Cardiol.50, 432-440 (2007).

10. Yao Y, Zhang S, He DS, et al. Radiofrequency ablation of the ventricular tachycardia with arrhythmogenic right ventricular cardiomyopathy using non-contact mapping. Pacing and Clinical Electrophysiology 30, 526-533 (2007). 